Published in "Soft Matter 14(19): 3978-3986, 2018"

which should be cited to refer to this work.

\title{
Against the rules: pressure induced transition from high to reduced order
}

\author{
Frederik Neuhaus, (D) ab Dennis Mueller, ${ }^{a}$ Radu Tanasescu, ${ }^{a}$ Cristina Stefaniu, ${ }^{c}$ \\ Pierre-Léonard Zaffalon, ${ }^{a}$ Sandor Balog, (D) d Takashi Ishikawa, (D) e Renate Reiter, \\ Gerald Brezesinski ${ }^{c}$ and Andreas Zumbuehl (iD *ab
}

\begin{abstract}
Envisioning the next generation of drug delivery nanocontainers requires more in-depth information on the fundamental physical forces at play in bilayer membranes. In order to achieve this, we combine chemical synthesis with physical-chemical analytical methods and probe the relationship between a molecular structure and its biophysical properties. With the aim of increasing the number of hydrogen bond donors compared to natural phospholipids, a phospholipid compound bearing urea moieties has been synthesized. The new molecules form interdigitated bilayers in aqueous dispersions and self-assemble at soft interfaces in thin layers with distinctive structural order. At lower temperatures, endothermic and exothermic transitions are observed during compression. The $\mathrm{LC}_{1}$ phase is dominated by an intermolecular hydrogen bond network of the urea moieties leading to a very high chain tilt of $52^{\circ}$. During compression and at higher temperatures, presumably this hydrogen bond network is broken allowing a much lower chain tilt of $35^{\circ}$. The extremely different monolayer thicknesses violate the two-dimensional Clausius-Clapeyron equation.
\end{abstract}

\section{Introduction}

The structure of a phospholipid bilayer membrane is based on the equilibrium of forces present between the different segments of a phospholipid: the hydrophobic tails, the hydrophilic headgroup, and the backbone connecting these segments. The classical packing parameter of a phospholipid molecule represents a local energy minimum taking into account the length of a hydrophobic fatty acyl chain, its volume and the area of the headgroup. ${ }^{1,2}$ Moving beyond a pure geometrical interpretation of the packing parameter, the attractive hydrophobic forces of the tails have to compensate the repulsive interactions between the headgroups. ${ }^{3}$ Additionally, the backbone, typically a glycerol moiety, is crucially important for the phase behaviour of a phospholipid bilayer: ${ }^{4}$ the tilt angle of the glycerol backbone

\footnotetext{
${ }^{a}$ Department of Chemistry, University of Fribourg, Chemin du Musée 9, 1700 Fribourg, Switzerland. E-mail: andreas.zumbuehl@unifr.ch

${ }^{b}$ National Center of Competence in Research in Chemical Biology, Quai Ernest Ansermet 30, 1211 Geneva, Switzerland

${ }^{c}$ Max Planck Institute of Colloids and Interfaces, Research Campus Potsdam-Golm, 14476 Potsdam, Germany

${ }^{d}$ Adolphe Merkle Institute, University of Fribourg, Chemin du Verdiers 4, 1700 Fribourg, Switzerland

${ }^{e}$ Paul Scherrer Institute (PSI), 5232 Villigen PSI, Switzerland

${ }^{f}$ Department of Experimental Polymer Physics, University of Freiburg,

Hermann Herder Strasse 3, 79104 Freiburg, Germany

$\dagger$ Electronic supplementary information (ESI) available. See DOI: 10.1039/c8sm00212f
}

significantly influences the packing parameter of the phospholipid by increasing the area of the headgroup compared to the in-plane area of the tails. The backbone orientation of a standard 1,2glycerophospholipid, such as 1,2-dimyristoyl-sn-glycero-3-phosphocholine, is nearly parallel to the bilayer membrane normal, ${ }^{5,6}$ whereas in dietherphospholipids, such as 1,2-dihexadecyl-phosphatidylcholine, the glycerol is roughly $45^{\circ}$ off the bilayer normal. ${ }^{7,8}$ The glycerol backbone is fully parallel to the bilayer when the substitution pattern is changed from the natural 1,2- to the artificial 1,3-pattern. ${ }^{9}$ If the increase of the headgroup area cannot be compensated anymore by acyl chain tilting (the threshold is below $60^{\circ}$ to the bilayer normal), the system will resolve to form an interdigitated bilayer membrane. ${ }^{10-12}$

Previously, we have reported about the artificial 1,3-diamidophospholipid Pad-PC-Pad. ${ }^{13-15}$ Because of the $s n-1,3$ constitution of the tails, the molecules form fully interdigitated bilayer membranes in the gel state. Vesicles formulated from Pad-PC-Pad show highly non-spherical geometries below $T_{\mathrm{m}} \cdot{ }^{13,15}$ This leads to membrane defects in the inner bilayer leaflet. ${ }^{13,16}$ Such defects are attenuated when the vesicles are exposed to high shear forces, and an entrapped vesicle cargo will be released. This led to the concept of mechano-responsive drug delivery. ${ }^{17-22}$ In order to fine-tune this concept, we have synthesized a new 1,3-diureaphospholipid: 1,3hexadecylurea-1,3-deoxy-sn-glycero-2-phosphatidylcholine, Sur-PCSur (1, see Scheme 1).

Mechano-responsive vesicles are facetted in the gel state. This is due to stiff bilayer membranes ensuing either from 

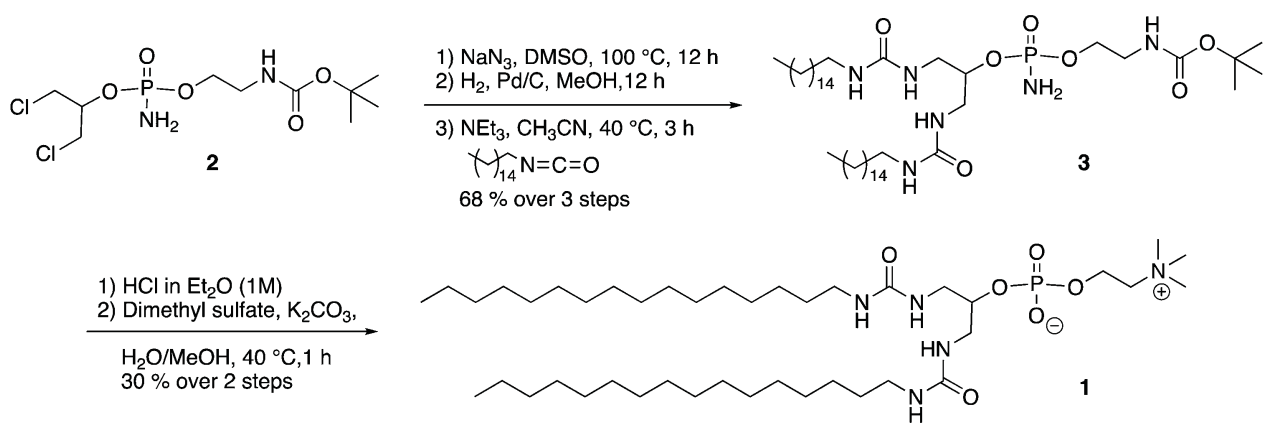

Scheme 1 Synthesis of Sur-PC-Sur (1) from dichlorophosphoramidate (2).

strong intermolecular forces ${ }^{23}$ or from membrane leaflet interdigitation. ${ }^{15}$ Sur-PC-Sur (1) is a perfect molecule to push the limits of both forces. Compared to Pad-PC-Pad, the chains were elongated by one methylene group each to increase the inter-chain hydrophobic forces, and the amides were replaced by urea moieties to increase the inter-bilayer hydrogen bonding capability. In analogy, long chain hydroxyethylureas form strong intermolecular hydrogen bonding networks. ${ }^{24}$ Expanding the concept to bis-tailed lipids without phosphocholine headgroups proved to be problematic, and an insoluble material has been obtained. ${ }^{25}$ We expected that the zwitterionic headgroup would mitigate the strong intermolecular forces and would again make it possible to formulate vesicles. Here, we report a detailed study of the monolayer and bilayer properties of the newly synthesized Sur-PC-Sur (1).

\section{Results and discussion}

The diureaphospholipid Sur-PC-Sur (1) was synthesized starting from the dichlorophosphoramidate 2 (see Scheme 1). ${ }^{26}$ Compound 2 was transferred into the diaminophosphoramidate using a modified Staudinger-type chemistry. Both amines were substituted with hexadecylisocyanate in order to yield the protected Sur-PE-Sur (3). According to our phospholipid nomenclature, S stands for stearoyl and ur stands for a urea substitution pattern at the lipid backbone. ${ }^{26}$ The order of the substituents corresponds to their position at the glycerol backbone: 1-Sur-2-PEBoc-3-Sur (3). Bocdeprotection, headgroup conversion to the ethanolamine and quaternization of the cephaline amine yielded the phosphocholine Sur-PC-Sur (1).

A hydrated powder sample of the bisurea compound Sur-PCSur (1) was analyzed by differential scanning calorimetry. Upon heating the phospholipid, one sharp main-phase transition $\left(T_{\mathrm{m}}\right)$ was found at $47.2{ }^{\circ} \mathrm{C}$ with an enthalpy change $\Delta H$ of $44.01 \mathrm{~kJ} \mathrm{~mol}^{-1}$ (see Fig. S7, ESI $\dagger$ ). Upon cooling, Sur-PC-Sur (1) showed a hysteresis and the $T_{\mathrm{m}}$ was found at $43.4{ }^{\circ} \mathrm{C}$ with $(\Delta H$ of $\left.48.64 \mathrm{~kJ} \mathrm{~mol}^{-1}\right)$. Such hysteresis is in line with data measured for hydrogen bonding ceramides. ${ }^{27,28}$ The $T_{\mathrm{m}}$ is below the value for the natural C18:0 DSPC $\left(55.3{ }^{\circ} \mathrm{C}\right)^{29}$ and its ethanolamine analog DSPE $\left(74.2^{\circ} \mathrm{C}\right) .{ }^{29}$ This hints at a higher hydration degree of the headgroup, as was to be expected when the backbone ester was exchanged by urea moieties. The $T_{\mathrm{m}}$ for Sur-PC-Sur (1) is also significantly lower than the value for the 1,3-diamidophospholipid Sad-PC-Sad $(\mathbf{1})\left(52{ }^{\circ} \mathrm{C}\right) .{ }^{14}$ The thermogram for Sur-PC-Sur (1) shows no pre-transition. The sharpness of the peaks stands for a highly cooperative phase transition. The hysteresis upon cooling of the sample means that the ordering of Sur-PC-Sur (1) from a liquid-crystalline into a gel phase is kinetically hindered, probably due to the unique organization of the gel phase. The powder spectrum of the hydrated Sur-PC-Sur (1) was measured at room temperature. The observed Bragg peaks in a ratio of $1: 2: 3$ indicate a lamellar structure with a $d$-value of only $4.8 \mathrm{~nm}$. The reason for such a small $d$-value could be either strong tilting of the acyl chains or an interdigitated structure. The latter is supported by the WAXS pattern with only one Bragg peak. The in-plane lattice is hexagonal indicating upright orientation of the chains, whereas chain tilting would lead to a lattice distortion. The observed in-plane area per acyl chain amounts to $19.8 \AA^{2}$ (see Fig. S8 and S9, ESI $\dagger$ ), a value which has been also found for DSPC (1,2-disteraroyl-phosphatidylcholine) in a gel phase with tilted chains, indicating that the interdigitation does not compulsorily lead to a tighter packing.

The cryo-transmission electron micrographs of extruded large unilamellar vesicles of Sur-PC-Sur (1, see Fig. S10-S12, ESI $\dagger$ ) were frozen from at room temperature, and it was found that most of the vesicles were disrupted and non-bicelle patches formed. This shows that compared to the 1,3-diamidophospholipid Pad-PC-Pad, the intermolecular forces in 1,3-diureaphospholipid appear too strong to consistently allow the formation of vesicles. Nonetheless, vesicles were found with a bimodal distribution of facetted and spherical ones. The facetted population had a bilayer membrane thickness of $4.77 \pm 0.80 \mathrm{~nm}$ (see Fig. S12, ESI $\dagger$ ), which is consistent with the formation of an interdigitated bilayer as seen in the powder SAXS/WAXS measurements. For the spherical vesicles, a membrane thickness of $6.87 \pm 1.04 \mathrm{~nm}$ was measured which is consistent with a non-interdigitated bilayer and a headgroup parallel to the membrane normal (see Fig. S11, ESI $\dagger$ ). ${ }^{30}$ Interestingly, the formulation of Sur-PC-Sur (1) in saline did not yield any vesicles.

An interesting property of non-spherical phospholipid vesicles is their mechano-responsiveness: ${ }^{13}$ the vesicles do not release an entrapped dye when they are left standing for a prolonged time. But upon vortex shaking, they do release significant amounts of the dye within a short period of time. Here, we encapsulated the self-quenching fluorescent dye 5(6)-carboxyfluorescein in high 
concentration in vesicles. For comparison, we added experiments performed with the natural phospholipid DPPC and the artificial phospholipid Pad-PC-Pad (see Fig. S13, ESI $\dagger$ ). ${ }^{13}$ The new Sur-PC-Sur (1) vesicles did indeed show mediocre mechanoresponsiveness: the vesicles remained tight over a week, but upon shaking the vesicles continuously released the entrapped dye, possibly via membrane defects that are transiently amplified by the application of shear forces during vortexing. After 1 min of vigorous shaking, $10 \%$ of the entrapped dye was released. This means that Sur-PC-Sur (1) will not be useful for mechanoresponsive delivery applications.

The Langmuir isotherms exhibit unusual temperature dependence. The lateral pressure of the main transition from the LE (liquid-expanded) to the LC (liquid-condensed) phase increases with increasing temperature as observed for other amphiphiles (see Fig. 1, bottom left). However, a second weak first-order transition can be seen at higher lateral pressures in a certain temperature range. This transition behaves unusual because the transition pressure decreases with increasing temperature. Between $21{ }^{\circ} \mathrm{C}$ and $22{ }^{\circ} \mathrm{C}$, there is a jump and the second transition merges with the first one. The molecular area above the second transition $\left(\mathrm{LC}_{2}\right)$ is much smaller compared to the one obtained at the same pressure but lower temperature $\left(\mathrm{LC}_{1}\right)$. GIXD experiments have been performed to illuminate the $2 \mathrm{D}$ structure of these two phases, and will be explained later. The slope in the $\mathrm{LC}_{1}$ phase between the $\mathrm{LE}$ and the second $\mathrm{LC}_{2}$ phase decreases continuously with increasing temperature. The compressional modulus $(K)$ of the $\mathrm{LC}_{1}$ phase decreases with increasing temperature. At $22{ }^{\circ} \mathrm{C}$ the compression modulus is so low, that the transition directly ends in the $\mathrm{LC}_{2}$ phase, which has an approximately constant compressional modulus over the range of measured temperatures.

In Fig. 1 (bottom, left), the phase transition pressure is plotted as a function of the temperature. The temperature dependence can be approximated by a linear function. The slope of the LE- $\mathrm{LC}_{1}$ transition amounts to $1.41 \mathrm{mN} \mathrm{m}^{-1} \mathrm{~K}^{-1}$, and that of the $\mathrm{LC}_{1}-\mathrm{LC}_{2}$ transition to $-1.65 \mathrm{mN} \mathrm{m}^{-1} \mathrm{~K}^{-1}$. The two transition lines do not meet each other because the $\mathrm{LC}_{1}-\mathrm{LC}_{2}$ transition disappears suddenly above $21{ }^{\circ} \mathrm{C}$. Using the two-dimensional Clausius-Clapeyron equation $(\mathrm{d} \pi / \mathrm{d} T=\Delta S / \Delta A)$, the transition entropy $\Delta S$ can be calculated (see Fig. 1, bottom right). The molecular area decreases during the transition in both cases: $A_{\mathrm{LE}}>$ $A_{\mathrm{LC} 1}$ and $A_{\mathrm{LC} 1}>A_{\mathrm{LC} 2}$, therefore, $\Delta A>0$ in both cases. However, $\mathrm{d} \pi / \mathrm{d} T$ has a different algebraic sign. This means that during compression the monolayer exhibits an exothermic and an endothermic transition. Such behaviour has never been observed before.

The slopes of the $\Delta S v s . T$ dependencies corresponding to the LE- $\mathrm{LC}_{1}$ and $\mathrm{LE}-\mathrm{LC}_{2}$ transitions are different. The extrapolation to $\Delta S=0$ leads to the critical temperature $T_{\mathrm{c}}$ above which no transition into a condensed state occurs. In the case of the LE- $\mathrm{LC}_{1}$ transition, $T_{\mathrm{c}}$ amounts to $\sim 302 \mathrm{~K}$, and for the $\mathrm{LE}-\mathrm{LC}_{2}$ transition to $\sim 311 \mathrm{~K}$. The endothermic $\mathrm{LC}_{1}-\mathrm{LC}_{2}$ transition yields also $\sim 311 \mathrm{~K}$. The difference of $10 \mathrm{~K}$ between the transition temperature in bulk $(320.4 \mathrm{~K})$ and the critical temperature of the
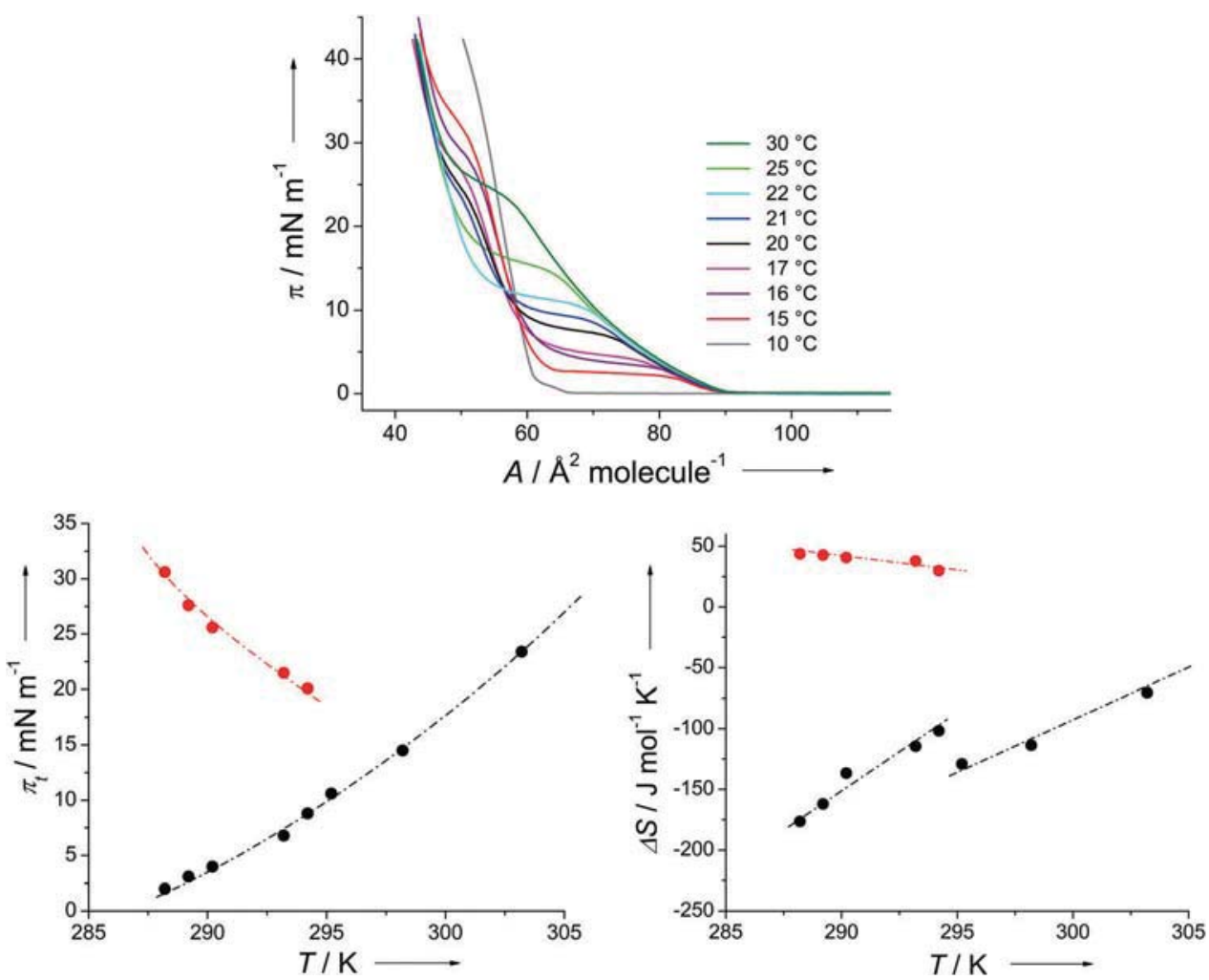

Fig. 1 (top) $\pi-\mathrm{A}$ isotherms of Sur-PC-Sur (1) monolayers on water and measured in the temperature range between $10{ }^{\circ} \mathrm{C}$ und $30{ }^{\circ} \mathrm{C}$. (bottom) Temperature dependence of the main phase transition pressure $\pi_{\mathrm{t}}$ (left) and the entropy change $\Delta S$ (right) of Sur-PC-Sur (1) monolayers on water during the transitions between LE and $\mathrm{LC}_{1}$ or $\mathrm{LC}_{2} \bullet$ (black) and between $\mathrm{LC}_{1}$ and $\mathrm{LC}_{2} \bigcirc$ (red). 

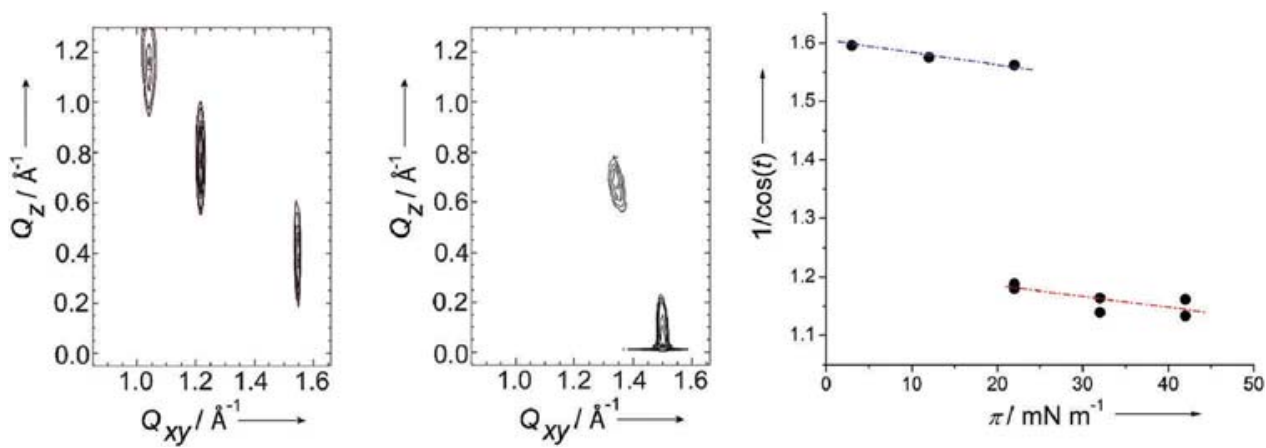

Fig. 2 Representative GIXD contour plots of the diffraction intensities as a function of the in-plane $Q_{x y}$ and out-of-plane $Q_{z}$ components of the scattering vector $Q$ for Sur-PC-Sur (1) in the $\mathrm{LC}_{1}$ phase at $10{ }^{\circ} \mathrm{C}$ and $3 \mathrm{mN} \mathrm{m}^{-1}$ (left) as well as in the $\mathrm{LC}_{2}$ phase at $20{ }^{\circ} \mathrm{C}$ and $30 \mathrm{mN} \mathrm{m}^{-1}$ (middle). Dependence of the tilt angle of the alkyl chains $(t)$ at $10{ }^{\circ} \mathrm{C}$ represented as $1 / \cos (t)$ on the lateral surface pressure $(\pi)$ (right).

monolayer $(311 \mathrm{~K})$ can be taken as an indication for differences in the in-plane packing modes in mono- and bilayers. ${ }^{14}$ This assumption is impressively supported by the fact that the gel phase of the bilayer system is an interdigitated one.

In Fig. 2, representative contour plots of the diffracted intensity versus the in-plane component $Q_{x y}$ and the out-of-plane component $Q_{z}$ of the scattering vector $Q$ are presented. Three Bragg peaks, indicating the in-plane packing of the chains into an oblique lattice structure, can be seen in the $\mathrm{LC}_{1}$ phase, whereas the $\mathrm{LC}_{2}$ phase is characterized by two Bragg peaks indicative of an orthorhombic packing of NN (nearest neighbour) tilted chains.

The striking differences between these two phases are the packing density and the tilt angle of the hydrocarbon chains. At low temperature $\left(10{ }^{\circ} \mathrm{C}\right)$, the $\mathrm{LC}_{1}$ phase is characterized by a cross-sectional area of only $19.4 \AA^{2}$. The strong headgroup interactions are very likely based on a hydrogen bond network. Since the headgroups need more space in the in-plane direction, the chains are strongly tilted to maximize their van der Waals interactions. A tilt angle above $50^{\circ}$ has been found. Compression practically does not change this large tilt angle (see Fig. 2, right). The transition pressure, at which the chains would become non-tilted, can be determined by extrapolation of $1 / \cos (t)$ to zero tilt angle. ${ }^{31}$ The obtained values $\left(340 \mathrm{mN} \mathrm{m}^{-1}\right.$ for $\mathrm{LC}_{1}$ and $120 \mathrm{mN} \mathrm{m}^{-1}$ for $\mathrm{LC}_{2}$ ) are physically absurd (much above the surface tension of pure water). The tilt angle in the $\mathrm{LC}_{1}$ phase decreases only by $0.5^{\circ}$ with increasing the temperature by $10 \mathrm{~K}$. In the $\mathrm{LC}_{2}$ phase, the temperature dependence is also not much pronounced (decrease of $1.5^{\circ}$ with increasing the temperature by $10 \mathrm{~K}$ ). The maximum tilt angle was determined by the extrapolation to zero surface pressure and amounts to $\sim 52^{\circ}$ in the $\mathrm{LC}_{1}$ and $\sim 35^{\circ}$ in the $\mathrm{LC}_{2}$ phase. At higher temperatures, the extrapolated tilting transition pressure is in a reasonable pressure range $\left(66.5 \mathrm{mN} \mathrm{m}^{-1}\right.$ at $20{ }^{\circ} \mathrm{C}$ and $45.4 \mathrm{mN} \mathrm{m}^{-1}$ at $24{ }^{\circ} \mathrm{C}$ ) (see Fig. S17 and S18, ESI $\dagger$ ).

According to the isotherm at $10{ }^{\circ} \mathrm{C}$, the monolayer is condensed at all pressures and no transitions can be seen, except the transition from the gas-analogues state to the $\mathrm{LC}_{1}$ phase (the transition pressure of the re-sublimation process is close to zero). During the long-lasting GIXD experiments, the $\mathrm{LC}_{1}$ phase transforms slowly into the $\mathrm{LC}_{2}$ phase. At $22 \mathrm{mN} \mathrm{m}^{-1}$, both phases coexist. This finding supports our assumption that the
$\mathrm{LC}_{1}$ phase transforms slowly into the $\mathrm{LC}_{2}$ phase even at continuous compression but elevated temperature. At lower temperatures, the transformation is obviously accelerated by keeping the lateral pressure constant (at least $30 \mathrm{~min}$ for each X-ray experiment), and maybe by illumination of the monolayer with the strong X-ray beam.

The lattice distortion $d$ versus $\sin ^{2}(t)$ is shown in Fig. S15 $(\mathrm{ESI} \dagger)$ for $10{ }^{\circ} \mathrm{C}^{32}$ The extrapolation to zero tilt allows to separate the contribution of the tilt from other contributions to the distortion such as backbone ordering. Fig. S15 (ESI $\dagger$ ) demonstrates that values obtained in phases $\mathrm{LC}_{1}$ and $\mathrm{LC}_{2}$ at $10{ }^{\circ} \mathrm{C}$ can only be fitted with two different linear functions. The intercept $d_{0}$ is clearly non-zero $(-0.40445)$ in the $\mathrm{LC}_{1}$ phase with much higher tilt angles and higher lattice distortion. This could be an indication of headgroup ordering preventing the hexagonal packing of the chains in the $\mathrm{LC}_{1}$ phase. In the $\mathrm{LC}_{2}$ phase, the intercept $d_{0}$ is much closer to zero (0.01415), and this value decreases with increasing temperature $\left(0.00252\right.$ at $20{ }^{\circ} \mathrm{C}$ and 0.00173 at $24{ }^{\circ} \mathrm{C}$ ) (see Fig. S19 and S20, ESI $\dagger$ ). Therefore, the tilt seems to be the only contribution to the distortion in the $\mathrm{LC}_{2}$ phase at higher temperatures.

The packing density decreases with increasing temperature (see Fig. S16, ESI $\dagger$ ). The cross-sectional area $A_{0}$ is very small in the $\mathrm{LC}_{1}$ phase. Such low value is an indication for a herringbone packing mode in agreement with the large lattice distortion. The $\mathrm{LC}_{2}$ phase has $A_{0}$ values typical for rotator phases, which increase with increasing temperature typical for the higher rotational mobility at higher temperatures.

In agreement with GIXD data, IRRA spectra recorded for Sur-PC-Sur (1) reveal a higher hydration degree of the phosphate group in the $\mathrm{LC}_{1}$ phase (Fig. $\mathrm{S} 21$ - blue line, $12{ }^{\circ} \mathrm{C}, 1 \mathrm{mN} \mathrm{m}^{-1}$ ) compared to the $\mathrm{LC}_{2}$ phase (Fig. S21 - red line, $20{ }^{\circ} \mathrm{C}$, $35 \mathrm{mN} \mathrm{m}^{-1}$, ESI $\dagger$ ). The $\mathrm{LC}_{1}$ phase defined by hydrogenbonded interconnected headgroups and high tilt angle of the alkyl chains offers large in-plane areas to the phosphocholine moieties which are highly hydrated. This is clearly depicted by the positions of the symmetric and antisymmetric $\mathrm{PO}_{2}^{-}$stretching vibration bands at 1086 and $1218 \mathrm{~cm}^{-1}$, respectively. For the $\mathrm{LC}_{2}$ phase, the positions of those bands are shifted to 1091 and $1227 \mathrm{~cm}^{-1}$, respectively. Such shift to higher values is characteristic for a dehydration effect of the phosphate moiety ${ }^{33-35}$ and can 
be well understood since the $\mathrm{LC}_{2}$ phase offers much less in-plane area to the headgroups compared to the $\mathrm{LC}_{1}$ phase. The $\mathrm{CH}_{2}$ stretching bands are at low wavenumbers, in accordance with the observed condensed phases. The amide bands are at the same positions in both condensed phases. The reason must be the fact that inter- and intramolecular hydrogen bonds cannot be distinguished by IRRAS.

The thickness of the Sur-PC-Sur (1) monolayer on water, compressed to $30 \mathrm{mN} \mathrm{m}^{-1}$, was determined by XRR at $20{ }^{\circ} \mathrm{C}$ ( $\mathrm{LC}_{2}$ phase). The obtained reflectivity curve and the best fit to the data are shown in Fig. 3. A model-free method was applied to convert the XRR data into the electron density profile (Fig. 3). This electron density can be satisfactorily modeled by a two-box model. The molecular area determined from the isotherm and the number of electrons in the hydrophobic part of the molecule were used as constrains during the fitting procedure. The hydrocarbon chain layer has a thickness $z$ of $17.6 \AA$ and the headgroup of $8.4 \AA$. Using a molecular area of $45 \AA^{2}$ and the obtained electron densities of $5.752 \mathrm{e}^{-} \AA^{-2}$ and $3.972 \mathrm{e}^{-} \AA^{-2}$, 259 electrons are in the chain region and 179 electrons in the headgroup region. The number of electrons in the headgroup region indicates that practically no water molecules are confined in a hydration shell around the headgroup in agreement with the IRRAS data, or cannot be seen because of no contrast to the surrounding water. The largest roughness $(4.8 \AA)$ is obtained between the headgroups and the water subphase, while the one between the chains and air is with 3.8 Å only $1 \AA$ A larger compared with that of a pure water surface.

As already several times verified, the use of a very simple model allows satisfying determination of $z=\left(\mathrm{MW}_{\text {chain }}\right) /\left(A \cdot \rho \cdot N_{\mathrm{A}}\right)$, with the molecular weight of the hydrophobic chains $\mathrm{MW}_{\text {chain }}$, the molecular area $A$ (determined from the isotherm), the density of the hydrophobic layer $\rho$ (the density of polyethylene $\left(0.9 \mathrm{~g} \mathrm{~cm}^{-3}\right)$ has been used as a first approximation), and the Avogadro constant $N_{\mathrm{A}}{ }^{36}$ The thicknesses of the hydrophobic layer amounts to $18.4 \AA$, in quite good agreement with the values determined from the reflectivity data. Assuming a stretched chain in all-trans conformation, the length can be calculated by $L_{\text {chain }}=(n 1.26+1.46)=20.4 \AA^{\circ} .^{37}$ The $1.26 \AA$ is the $\mathrm{C}-\mathrm{C}$ distance $(\mathrm{C}-\mathrm{C}$ bond length of $1.54 \AA$ and bond angle $109.5^{\circ}$ ) projected on the all-trans chain axis, and $1.46 \AA$ takes the influence of the $\mathrm{CH}_{3}$ group into account. Comparing the thickness (averaged value of $18 \AA$ ) with the length of the chains leads to a tilt angle of $28^{\circ}$. This value is in very good agreement with the tilt angle determined by GIXD experiments (see in ESI $\dagger$ Tables S1-S3).

Comparing the structural properties of the $\mathrm{LC}_{1}$ and $\mathrm{LC}_{2}$ phases with the transition entropy, obtained by the twodimensional Clausius-Clapeyron equation, reveals an apparent discrepancy. At two similar temperatures $\left(21\right.$ and $\left.22{ }^{\circ} \mathrm{C}\right)$, the transition from the LE phase to the condensed phase state leads to the densely packed and strongly tilted $\mathrm{LC}_{1}$ phase or to the rotator phase $\mathrm{LC}_{2}$, respectively. The transition pressure (see Fig. 1) increases continuously with increasing temperature. However, the negative transition entropy exhibits a jump to larger absolute values $\left(-101.8 \mathrm{~J} \mathrm{~mol}^{-1} \mathrm{~K}^{-1}\right.$ at $21{ }^{\circ} \mathrm{C}$ and $-128.9 \mathrm{~J} \mathrm{~mol}^{-1} \mathrm{~K}^{-1}$ at $22{ }^{\circ} \mathrm{C}$ ) because of the smaller in-plane area of phase $\mathrm{LC}_{2}$ compared to $\mathrm{LC}_{1}$. Assuming similar entropy values in the liquid-like LE phase at similar temperatures, one would expect smaller entropy values in the tighter packed $\mathrm{LC}_{1}$ phase and hence the opposite trend in the $\Delta S$ values. The problem is obviously the misleading use of the two-dimensional Clausius-Clapeyron equation because of the drastically different thicknesses between $\mathrm{LC}_{1}$ and $\mathrm{LC}_{2}$. In the present case, the change in molecular area $\left(\Delta A_{\mathrm{LE}-\mathrm{LC} 1}=\right.$ $-12 \AA^{2}$ molecule ${ }^{-1}$ and $\Delta A_{\mathrm{LE}-\mathrm{LC} 2}=-15.2 \AA^{2}$ molecule $\left.^{-1}\right)$ has to be replaced by the change in molecular volume. Taking into account only the thicknesses of the hydrophobic part, we end up with the following numbers: $L_{\mathrm{LE}} \sim 15 \AA, L_{\mathrm{LC} 1} \sim 13 \AA$ (with a tilt angle of $50^{\circ}$ ), $L_{\mathrm{LC} 2} \sim 18 \AA$ (with a tilt angle of $30^{\circ}$ ). These numbers lead to $\Delta V$ values of $-290 \AA^{3}$ molecule $^{-1}\left(\mathrm{LE}-\mathrm{LC}_{1}\right)$ and $-87.3 \AA^{3}$ molecule $^{-1}\left(\mathrm{LE}-\mathrm{LC}_{2}\right)$. This results in $\Delta A_{\mathrm{LE}-\mathrm{LC} 1} /$ $\Delta A_{\mathrm{LE}-\mathrm{LC} 2}=0.79$ and $\Delta V_{\mathrm{LE}-\mathrm{LC} 1} / \Delta V_{\mathrm{LE}-\mathrm{LC} 2}=3.32$, and the jump in
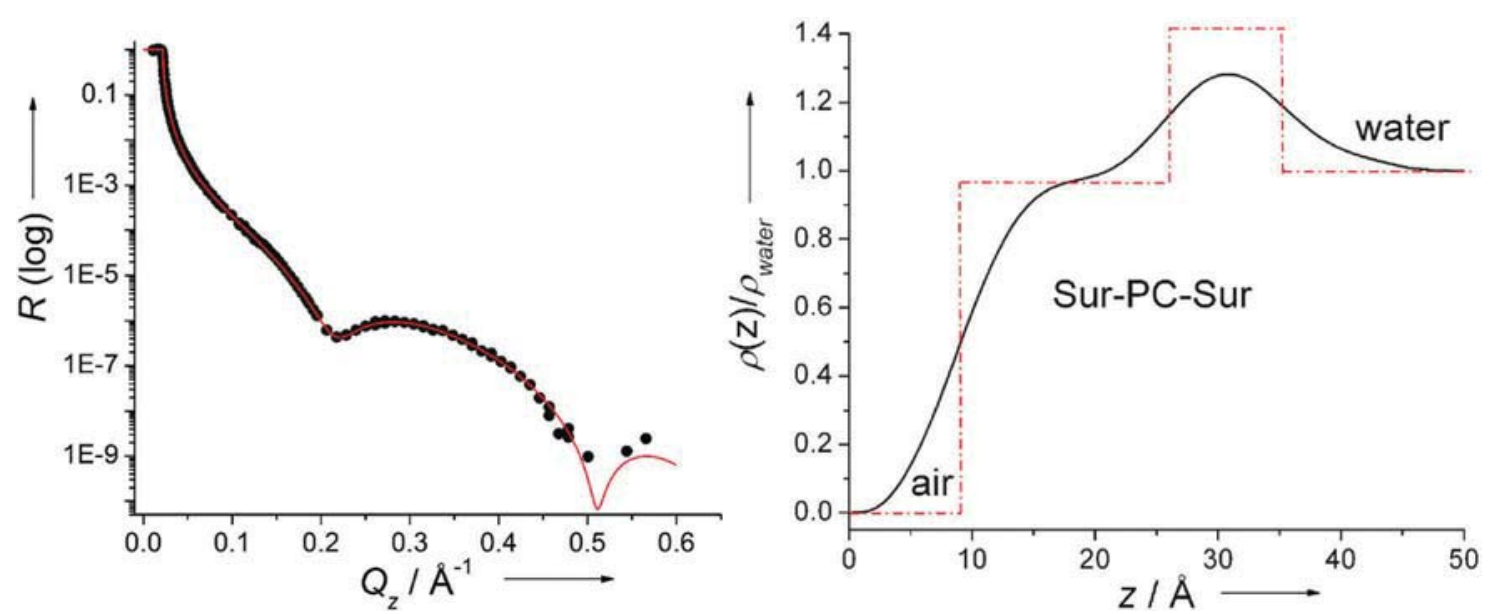

Fig. 3 (left) Specular X-ray reflectivity of Sur-PC-Sur (1) at $20^{\circ} \mathrm{C}$ on water at $30 \mathrm{mN} \mathrm{m}^{-1}$ (dots). The calculated reflectivity is shown as red line. (right) The electron density normalized by the electron density of water versus depth $z$. The applied box-model is shown (red dotted-dashed line). Comparing the hydrocarbon layer thickness with the theoretical length of the chains leads to a tilt angle of $28^{\circ}$. This value is in very good agreement with the tilt angle determined by GIXD experiments. 
$\Delta S$ is clearly contrary to what was obtained by using the twodimensional Clausius-Clapeyron equation even if the surface pressure has still to be transformed into the $3 \mathrm{D}$ pressure. This opposite jump is now in perfect agreement with the structural data, and demonstrates that the use of the two-dimensional Clausius-Clapeyron equation is not recommended in case of phase transitions connected with extreme changes in monolayer thicknesses.

Brewster-angle microscopy was used in order to visualize the two different condensed phases $\mathrm{LC}_{1}$ and $\mathrm{LC}_{2}$ (see Fig. S22, ESI $\dagger$ ). The nucleation process in the two-phase coexistence region during the first-order transition from $\mathrm{LE}$ to either $\mathrm{LC}_{1}$ or $\mathrm{LC}_{2}$ yielded too small domains (high nucleation rate and low growth rate) to draw any conclusion about differences in the condensed phases. Above the plateau, $\mathrm{LC}_{1}$ is characterized by ribbons of two coexisting phases with different thickness (different brightness of the ribbons). This seems to support our observations during GIXD experiments that the $\mathrm{LC}_{1}$ phase transforms slowly into the $\mathrm{LC}_{2}$ phase. These two phases have strongly different thicknesses. At higher temperatures, the $\mathrm{LC}_{2}$ phase, formed directly from LE, is much more homogeneous with an almost uniform surface coverage of the subphase. These data are in accordance with the above described observations.

\section{Experimental}

\section{General information}

The starting compounds and solvents were purchased from Sigma-Aldrich/Fluka, ABCR, TCI Chemicals or Acros and were used without further purification. For reactions under inert gas conditions, the solvents (dichloromethane and chloroform) were dried over molecular sieves $4 \AA$ and degassed afterwards. Column chromatography was carried out using 230-400 mesh, 60 Å silica gel (Brunschwig). TLC plates (Merck, Silica gel $60 \AA$ F254) were developed with $\mathrm{KMnO}_{4}$-solution. ${ }^{1} \mathrm{H},{ }^{13} \mathrm{C}$ and ${ }^{31} \mathrm{P}$ NMR spectra were recorded (as indicated) on either a Bruker 300 or $400 \mathrm{MHz}$ spectrometer and are reported as chemical shifts in ppm relative to the signal of the deuterated NMR-solvent. Spin multiplicities are reported as singlet (s), doublet (d), triplet $(\mathrm{t})$, with coupling constants $(J)$ given in $\mathrm{Hz}$, or multiplets $(\mathrm{m})$. Broad peaks are marked as br. HRESI-MS was performed on a QSTAR Pulsar (AB/MDS Sciex) spectrometer and are reported as massper-charge ratio $m / z$. IR spectra were recorded on a PerkinElmer Spectrum One FT-IR spectrometer (ATR, Golden Gate).

tert-Butyl(2-((amino((1,3-dichloropropan-2-yl)oxy)phosphoryl)oxy)ethyl)carbamate (2). Following our literature procedure, ${ }^{26}$ $\mathrm{NEt}_{3}(7.0 \mathrm{~mL}, 494.1 \mathrm{mg}, 50.0 \mathrm{mmol})$ was added to a solution of $\mathrm{POCl}_{3}(5.9 \mathrm{~mL}, 7.7 \mathrm{~g}, 50.0 \mathrm{mmol})$ in $\mathrm{Et}_{2} \mathrm{O}(120 \mathrm{~mL})$. Then the reaction was cooled to $0{ }^{\circ} \mathrm{C}$ and 1,3-dichloro-2-propanol $(4.8 \mathrm{~mL}$, $6.5 \mathrm{~g}, 50.0 \mathrm{mmol}$ ) was added dropwise. The reaction mixture was stirred for $4.75 \mathrm{~h}$. The mixture was filtered and washed with $\mathrm{Et}_{2} \mathrm{O}$ $(3 \times 40 \mathrm{~mL})$. The solvent was removed under reduced pressure to obtain a lightly yellow oil, which was used without further purification. The oil was dissolved in DCM $(80 \mathrm{~mL}), \mathrm{NEt}_{3}$ (7.0 mL, $5.1 \mathrm{mg}, 50.0 \mathrm{mmol}$ ) was added and afterwards the mixture was cooled to $0{ }^{\circ} \mathrm{C} . \mathrm{N}$-Boc-ethanolamine $(8.1 \mathrm{mg}$, $7.7 \mathrm{~mL}, 50 \mathrm{mmol})$ dissolved in DCM $(20 \mathrm{~mL})$ was added dropwise during $2 \mathrm{~h}$. After the addition, the reaction mixture was stirred for further $4.5 \mathrm{~h}$ at RT. The reaction mixture was quenched with $\mathrm{NH}_{4} \mathrm{OH}(90 \mathrm{~mL}, 25 \%)$ at $0{ }^{\circ} \mathrm{C}$ and afterwards it was stirred for further $30 \mathrm{~min}$ at RT. The phases were separated and the organic layer was washed with saturated $\mathrm{NH}_{4} \mathrm{Cl}(40 \mathrm{~mL})$. The aqueous layer was extracted with DCM $(3 \times 40 \mathrm{~mL})$. The combined organic layers were dried over $\mathrm{Na}_{2} \mathrm{SO}_{4}$ and concentrated under reduced pressure. The product was purified by silica gel column chromatography $\left(\mathrm{SiO}_{2}, \mathrm{EtOAc}\right)$. A white powder was obtained (7.35 g, $20.9 \mathrm{mmol}, 42 \%)$.

${ }^{1} \mathrm{H}-\mathrm{NMR}:\left(300 \mathrm{MHz} ; \mathrm{CDCl}_{3}, \delta\right): 5.06(\mathrm{~s} \mathrm{br}, 1 \mathrm{H}), 4.77-4.67(\mathrm{~m}$, $1 \mathrm{H})$, 4.19-4.04 (m, 2H), 3.88-3.76 (m, 4H), 3.51-3.33 (m, $2 \mathrm{H})$, $3.11(\mathrm{~d}, J=4.8 \mathrm{~Hz}, 2 \mathrm{H}), 1.43(\mathrm{~s}, 9 \mathrm{H})$.

${ }^{31}$ P-NMR: (121 MHz; $\left.\mathrm{CDCl}_{3}, \delta\right): 9.1$.

$R_{\mathrm{f}}$ (EtOAc): 0.27 .

tert-Butyl(2-((amino((1,3-bis(3-hexadecylurea)propan-2-yl)oxy)phosphoryl)oxy)ethyl)carbamate (3). The dichlorophosphoramidite 2 (500.1 mg, $1.43 \mathrm{mmol}$ ) was dissolved in DMSO (35 mL) and $\mathrm{NaN}_{3}$ (250 $\mathrm{mg}, 3.83 \mathrm{mmol}$ ) was added and the resulting solution was heated to $100{ }^{\circ} \mathrm{C}$ overnight. Afterwards it was cooled to RT. Brine $(30 \mathrm{~mL})$ was added and the solution was extracted with $\mathrm{Et}_{2} \mathrm{O}$ $(25 \mathrm{~mL})$. The aqueous phase was washed with $\mathrm{Et}_{2} \mathrm{O}(4 \times 25 \mathrm{~mL})$. The combined organic phases were dried over $\mathrm{Na}_{2} \mathrm{SO}_{4}$. $\mathrm{MeOH}$ $(50 \mathrm{~mL})$ was added and $\mathrm{Et}_{2} \mathrm{O}$ was removed under reduced pressure. The crude diazide, still solved in $\mathrm{MeOH}$, was used without further purification. Palladium on charcoal $(75 \mathrm{mg})$ was added to the solution of the crude product in $\mathrm{MeOH} . \mathrm{H}_{2}$ was bubbled through the solution (one filled balloon). Afterwards a second balloon filled with $\mathrm{H}_{2}$ was added and the solution was stirred overnight. To get rid of the Pd-catalyst the solution was filtered over celite and washed with $\mathrm{MeOH}(3 \times 30 \mathrm{~mL})$. The solvent was removed under reduced pressure. The resulting crude diamine was used without further purification. The crude product was solved in acetonitrile $(20 \mathrm{~mL})$ and $\mathrm{NEt}_{3}(5 \mathrm{~mL}$, $3.63 \mathrm{~g}, 35.8 \mathrm{mmol}$ ) was added and the mixture was heated to $40{ }^{\circ} \mathrm{C}$. Afterwards hexadecylisocyanate $(765 \mathrm{mg}, 0.89 \mathrm{~mL}$, $2.86 \mathrm{mmol}$ ) was added slowly and it was stirred for $3 \mathrm{~h}$ at $40{ }^{\circ} \mathrm{C}$. After cooling to room temperature, the reaction mixture was filtered and the filtrate was washed with DCM $(30 \mathrm{~mL})$. The solvents were removed under reduced pressure and the crude product was purified by column chromatography (silica gel; EtOAc/MeOH, 9:1, v:v). The product was isolated as a white wax (825.0 mg, $0.97 \mathrm{mmol}, 68 \%)$.

${ }^{1} \mathrm{H}-\mathrm{NMR}:\left(300 \mathrm{MHz} ; \mathrm{CDCl}_{3}, \delta\right.$ ): 6.11 (s br, 1H), 6.03 (s br, $1 \mathrm{H}$ ), $5.70(\mathrm{~s} \mathrm{br}, 1 \mathrm{H}), 5.38(\mathrm{~s} \mathrm{br}, 1 \mathrm{H}), 5.17(\mathrm{~s}, 1 \mathrm{H}), 4.31(\mathrm{~s} \mathrm{br}, 1 \mathrm{H})$, 4.15-4.00 (m, 4H), 3.90-3.89 (m, 1H), 3.37-3.32 (m, 4H), 3.14-3.07 (m, 4H), $1.43(\mathrm{~s}, 13 \mathrm{H}), 1.24(\mathrm{~s}, 52 \mathrm{H}), 0.89(\mathrm{t}, 6 \mathrm{H})$.

${ }^{31}$ P-NMR: (121 MHz; $\left.\mathrm{CDCl}_{3}, \delta\right): 10.2$.

Sur-PC-Sur (1). N-Boc-protected Sur-PE-Sur 3 (1.67 g, $2.11 \mathrm{mmol}$ ) was dissolved in $\mathrm{HCl}$ in $\mathrm{Et}_{2} \mathrm{O}(1 \mathrm{M}, 15 \mathrm{~mL})$ plus a few drops of water. It was stirred for $1.5 \mathrm{~h}$ at room temperature. The solvents were removed under reduced pressure. Sur-PE-Sur was dissolved in MeOH (30 mL). Dimethyl sulfate (1.45 mL, $1.93 \mathrm{~g}, 15.3 \mathrm{mmol}$ ) was added and the mixture was heated up to $40{ }^{\circ} \mathrm{C}$. $\mathrm{A} \mathrm{K}_{2} \mathrm{CO}_{3}$ solution 
(2.11 g, $15.3 \mathrm{mmol}$, in $20 \mathrm{~mL}$ water) was added and the mixture was stirred at $40{ }^{\circ} \mathrm{C}$ for $1 \mathrm{~h}$. After cooling down to room temperature the solvents were removed under reduced pressure, the crude product was purified by column chromatography (silica, DCM $/ \mathrm{MeOH} / \mathrm{H}_{2} \mathrm{O}, 65: 25: 4$ ) and precipitated from $\mathrm{CHCl}_{3} / \mathrm{MeOH}(1: 1)$ with acetone. A white powder was obtained (500 mg, $0.63 \mathrm{mmol}, 30 \%$ ).

${ }^{1} \mathrm{H}$ NMR $\left(400 \mathrm{MHz}, \mathrm{CDCl}_{3}, \delta\right): 6.51(\mathrm{NH}, \mathrm{br} \mathrm{s}, 2 \mathrm{H}), 5.82(\mathrm{NH}$, br s, $2 \mathrm{H}), 4.35\left(\mathrm{CH}_{2}\right.$, br s, $\left.2 \mathrm{H}\right), 4.11(\mathrm{CH}$, br s, $1 \mathrm{H}), 3.82\left(\mathrm{CH}_{2}\right.$, br s, $2 \mathrm{H}), 3.42-3.35\left(\mathrm{CH}_{2}, \mathrm{~m}, 2 \mathrm{H}\right), 3.30\left(\mathrm{CH}_{3}, \mathrm{~s}, 9 \mathrm{H}\right), 3.21(\mathrm{~d}, J=7.1$ $\mathrm{Hz}, 2 \mathrm{H}), 3.05\left(\mathrm{CH}_{2}\right.$, br s, $\left.4 \mathrm{H}\right), 1.46-1.36\left(\mathrm{CH}_{2}, \mathrm{~m}, 4 \mathrm{H}\right), 1.25\left(\mathrm{CH}_{2}\right.$, s, 52), $0.87\left(\mathrm{CH}_{3}, \mathrm{t}, J=6.7 \mathrm{~Hz}, 6 \mathrm{H}\right)$.

${ }^{13} \mathrm{C} \quad \mathrm{NMR} \quad\left(100 \mathrm{MHz}, \quad \mathrm{CDCl}_{3}, \delta\right): \quad 159.5, \quad 54.4, \quad 50.7$, $40.6,32.1,30.8,29.9,29.9,29.9,29.9,29.8,29.7,29.5,27.3$, $22.8,14.3$.

${ }^{31} \mathrm{P}$ NMR $\left(162 \mathrm{MHz}, \mathrm{CDCl}_{3}, \delta\right):-1.3$.

HRMS $(m / z):\left[M+\mathrm{H}^{+}\right]$calcd for $[\mathrm{M}+\mathrm{H}], 790.6550$; found, 790.6541.

IR: 2916, 2849, 1634, 1564, 1466, 1209, 1056, 1003, 755, $579 \mathrm{~cm}^{-1}$. $R_{\mathrm{f}}\left(\mathrm{DCM} / \mathrm{MeOH} / \mathrm{H}_{2} \mathrm{O}, 65: 25: 4, \mathrm{v}: \mathrm{v}: \mathrm{v}\right): 0.67$.

\section{Differential scanning calorimetry}

Adopted from ref. 23. Multilamellar vesicle suspensions were prepared by hydration of $1 \mathrm{mg}$ of phospholipid powder with $1 \mathrm{~mL}$ of ultra-pure water (18.2 $\mathrm{M} \Omega \mathrm{m})$. The unextruded vesicle suspensions were degassed for 30 minutes using a TA degassing station. The alternating heating-cooling scans were recorded from $5{ }^{\circ} \mathrm{C}$ to $90{ }^{\circ} \mathrm{C}$ with a scanning speed of $0.5{ }^{\circ} \mathrm{C} \mathrm{min}^{-1}$. The experiment was performed twice, starting with new suspensions, in order to ensure reproducibility. The scans of the second heatingcooling scans are reported. Raw data was baseline corrected and converted to molar heat capacity (MHC) using the NanoAnalyze software (TA Instruments, USA). The main phase transition and enthalpy were determined with the same software and verified using the OriginLab software (OriginLabs, USA).

\section{Small-angle and wide-angle X-ray scattering}

A lipid dispersion (MLV, $20 \mathrm{wt} \%$ in ultra-pure water) was transferred into glass capillaries (inner diameter $2 \mathrm{~mm}$, GLAS, Germany). The small angle X-ray scattering (SAXS) was carried out at the in-house pinhole Instrument with rotating anode. The diffracted signal has been measured with a Mar CCD plate detector (Evanston, Illinois, USA). The incoming beam had a wavelength of $0.154 \mathrm{~nm}$, and the exposure time was $2 \mathrm{~h}$. The temperature was fixed at $21{ }^{\circ} \mathrm{C}$ during measurements. Positions of the Bragg peaks were converted into real space repeat distances of the lattice planes. In order to determine peak maxima and the full-width at half-maximum (FWHM) Lorentzian curves have been fitted to the experimental points. Correlation lengths $\xi$ were calculated using eqn (1) of the first order diffraction peak.

\section{Vesicle preparation}

Adapted from ref. 23. LUVET $_{100}$ of Sur-PC-Sur (1) were prepared following the standard extrusion protocol: in a $25 \mathrm{~mL}$ round bottom flask, $10 \mathrm{mg}$ of the lipid were dissolved in $\mathrm{CH}_{2} \mathrm{Cl}_{2}$. After evaporation of the organic solvent, the film was further dried under high vacuum (40 mbar) overnight. Then the film was hydrated with the internal buffer for $30 \mathrm{~min}$ (1 mL, $50 \mathrm{mM} 5(6)-$ carboxyfluorescein, $10 \mathrm{mM}$ HEPES buffer (AppliChem), $10 \mathrm{mM}$ $\mathrm{NaCl}$ dissolved in ultra-pure water, $\mathrm{pH} 7.4(\mathrm{NaOH}))$. Now at least five freeze-thaw cycles (liquid $\mathrm{N}_{2}$ to $65^{\circ} \mathrm{C}$ ) were carried out before the suspension was extruded 11 times using a Mini Extruder (Avanti Polar Lipids, USA) using track-edged filters with a mesh size of $100 \mathrm{~nm}$ (Whatman, USA). The external buffer was then exchanged on a size exclusion column $(1.5 \times 20 \mathrm{~cm}$ Sephadex G-50 column) in external buffer (107 mM NaCl, $10 \mathrm{mM}$ HEPES dissolved in ultra-pure water, $\mathrm{pH} 7.4(\mathrm{NaOH}))$.

\section{Cryogenic transmission electron microscopy}

Adapted from ref. 14. LUVET $_{100}$ of Sur-PC-Sur (1) were prepared by the extrusion protocol described above at a concentration of $10 \mathrm{mg} \mathrm{mL} \mathrm{m}^{-1}$. The liposome suspensions were let to reach room temperature and were diluted $1: 1$ with isotonic saline and were mounted on glow-discharged holey carbon grids, quickly frozen by a Cryoplunge 3 system (Gatan, USA) and transferred to a JEM2200FS transmission electron microscope (JEOL, Japan) using a Gatan626 cryo-holder. Cryo-electron micrographs were recorded at the acceleration voltage of $200 \mathrm{kV}, \times 20000$ magnification, 4-8 $\mu \mathrm{m}$ underfocus and a dose of 10 electrons per $\AA^{2}$, using a F416 CMOS detector (TVIPS, Germany). The final concentration of the liposomal suspension used was $5 \mathrm{mg} \mathrm{mL}^{-1}$ or $6.3 \mathrm{mM}$. The thickness of the bilayer was measured using the open source software Fiji. ${ }^{38-40}$

\section{Release experiments}

Adapted from ref. 23. The inner buffer (CF buffer) was prepared from $50 \mathrm{mM}$ 5(6)-carboxyfluorescein (powder, Sigma-Aldrich) and $10 \mathrm{mM}$ HEPES buffer (powder, Sigma-Aldrich) dissolved in ultra-pure water $(18.2 \mathrm{M} \Omega \mathrm{cm})$. The $\mathrm{pH}$ was adjusted to 7.4 $(\mathrm{NaOH})$ and the osmotic concentration to $200 \mathrm{mOs} \mathrm{mL}^{-1}(\mathrm{NaCl})$. The outer buffer was prepared from $10 \mathrm{mM}$ HEPES buffer (powder, Sigma-Aldrich) dissolved in ultra-pure water $(18.2 \mathrm{M} \Omega \mathrm{cm})$. The $\mathrm{pH}$ was adjusted to $7.4(\mathrm{NaOH})$ and the osmotic concentration to $200 \mathrm{mOs} \mathrm{mL}^{-1}$ (NaCl). Sur-PC-Sur (1) (2 mg) was weighed into a round-bottomed flask $(25 \mathrm{~mL})$ and dissolved in $\mathrm{CHCl}_{3}(1 \mathrm{~mL}$, amylene stabilized, Sigma-Aldrich, USA). The solvent was removed by low-pressure rotatory evaporation. The thin film was dried under high vacuum overnight, to ensure the removal of residual water and prevent cholesterol oxidation. Inner buffer $(1 \mathrm{~mL})$ was added to the round-bottomed flask and the film was hydrated for 30 minutes at $65{ }^{\circ} \mathrm{C}$. The film was subjected to five cycles of freeze/thaw using liquid nitrogen and a $65{ }^{\circ} \mathrm{C}$ water bath. The resulting MLV suspension was extruded 11 times through a track-etched filter membrane at $65{ }^{\circ} \mathrm{C}(100 \mathrm{~nm}$, Whatman, USA) placed in a Mini Extruder (Avanti Polar Lipids, USA). The vesicles were left standing at room temperature overnight. The residual non-encapsulated CF buffer, in the LUVET $_{100}$ suspension, was exchanged with the outer buffer using size exclusion chromatography (PD-10 desalting columns, GE Healthcare, UK). The size exclusion chromatography was carried out after 24 hours storage, in the dark at room temperature, of the LUVET $_{100}$ suspension. The purified LUVET ${ }_{100}$ suspension 
was diluted, in a volumetric flask, to $100 \mathrm{~mL}$ using additional outer buffer. Six aliquots $(2 \mathrm{~mL})$ were separated into vials $(5 \mathrm{~mL}$, PE caps) and vortex mixed for different amounts of time (0, 5 , $10,20,30,60 \mathrm{~s}$ ) at $2500 \mathrm{rpm}$. The 5(6)-carboxyfluorescein release was quantified using a fluorospectrometer (Sense 425-301, Hidex, Finland). For each sample, five microplate wells were filled with $200 \mu \mathrm{L}$ of the vortex 20 mixed vesicular suspension. The wavelengths used for measurements were $485 \mathrm{~nm}$ (excitation) and $535 \mathrm{~nm}$ (emission). As a control for the maximum dye release (F100), a Triton X-100 solution ( $2 \mu \mathrm{L}$ of a $10 \mathrm{vol} \%$ solution) was added to additional five microplate wells, filled with $200 \mu \mathrm{L}$ of vesicular suspension, for each sample. The release fraction at time $X$ was calculated with the formula: release $(\%)=\left(F_{X}-F_{0}\right) /\left(F_{100}-F_{0}\right)$, where $F_{X}$ is the fluorescence at time $X, F_{0}$ the fluorescence at time zero and $F_{100}$ the maximum fluorescence recorded after treatment with Triton X-100.

\section{Film balance measurements}

Adapted from ref. 23. Pressure-area isotherms were recorded on a computer interfaced Langmuir trough with a surface area of $194 \mathrm{~cm}^{2}$ (Riegler \& Kierstein, Potsdam, Germany). The paper plate Wilhelmy method was used to measure the surface tension with an accuracy of $\pm 0.1 \mathrm{mN} \mathrm{m}^{-1}$. Each measurement was repeated at least three times. The Langmuir trough was filled with ultrapure water (specific resistance of $18.2 \mathrm{M} \Omega \mathrm{cm}$ ). The lipid solution $\left(1 \mathrm{mg} \mathrm{mL} \mathrm{m}^{-1}\right)$ in chloroform was spread and the measurement was started 5-15 min after spreading.

\section{Grazing incidence X-ray diffraction measurements}

Adapted from ref. 14. The lattice structure of the monolayers was investigated using the liquid surface diffractometer at the undulator beamline BW1 (HASYLAB, DESY, Hamburg, Germany) by grazing incidence X-ray diffraction (GIXD) measurements. At BW1, a Langmuir film balance equipped with a Wilhelmy plate was positioned in a hermetically closed container flushed with helium. A monochromatic synchrotron X-ray beam $(\lambda=1.304 \AA)$ was adjusted to strike the helium/water interface at a grazing incidence angle $\alpha_{i}=0.85 \alpha_{c}\left(\alpha_{c}=0.13^{\circ}\right)$ lighting up roughly $2 \times$ $50 \mathrm{~mm}^{2}$ of the surface. The trough was laterally moved during the measurements to prevent any sample damage by the strong X-ray beam. For the measurement of the diffracted signal a MYTHEN detector system (PSI, Villigen, Switzerland) was rotated to scan the in-plane $Q_{x y}$ component values of the scattering vector. The vertical strips of the MYTHEN measured the out-of-plane $Q_{z}$ component of the scattering vector between 0.0 and $0.75 \AA^{-1}$. The diffraction data consisted of Bragg peaks at diagnostic $Q_{x y}$ values. The diffracted intensity normal to the interface was integrated over the $Q_{x y}$ window of the diffraction peak to calculate the corresponding Bragg rod.

\section{Infrared reflection-absorption spectroscopy}

Adapted from ref. 14. Infrared reflection-absorption spectra were recorded using the Vertex 70 FT-IR spectrometer (Bruker, Germany), equipped with a liquid-nitrogen cooled MCT detector and coupled to a Langmuir film balance, which was placed in a sealed container (an external air/water reflection unit
(XA-511, Bruker)) to guarantee a constant vapor atmosphere. Using a KRS-5 (thallium bromide and iodide mixed crystal) wire grid polarizer, the IR-beam was polarized parallel (p) or vertical (s) and focused on the fluid subphase at an angle of incidence of $40^{\circ}$. A computer controlled "trough shuttle system" enables us to choose between the compartment with the sample (subphase with spread layer) and a reference compartment (pure subphase). The single-beam reflectance spectrum from the reference trough was taken as background for the single-beam reflectance spectrum of the monolayer in the sample trough to calculate the reflection absorption spectrum as $-\log \left(R / R_{0}\right)$ in order to eliminate the water vapor signal. FTIR spectra were collected at a resolution of $8 \mathrm{~cm}^{-1}$ using 200 scans for s-polarized light and 400 scans for p-polarized light.

\section{Brewster-angle microscopy}

Adapted from ref. 41. Brewster angle micrographs were recorded with a commercially available Multiskop (Optrel, Germany) equipped with a Langmuir trough from Riegler \& Kierstein constructed identically to the one described above. Great care was taken that the investigated films were prepared under the same experimental conditions as for the monolayers that have already been discussed in this article. Parallel polarized laser light with respect to the plane of incidence of a wavelength of $\lambda=632.8 \mathrm{~nm}$ is reflected on the water surface under the Brewster angle of water $\left(53.1^{\circ}\right)$. The polarizer and analyzer were set to $0^{\circ}$ to suppress reflected light from a pure water surface. The reflected beam, which maps the inhomogeneities of the Langmuir film, is focused on a CCD camera for visualization. An objective of 10-fold magnification (Mitutoyo) is mounted on a piezo translation stage that moves continuously to bring consecutive stripes of the image into focus. A streaming video sequence is recorded to construct an overall sharp image. The lipid films were imaged at different stages of monolayer compression.

\section{Conclusion}

A new phospholipid has been synthesized with an increased number of hydrogen bond donors at the hydrophilic/hydrophobic interface (Sur-PC-Sur (1)). Monolayer experiments show two condensed phases and for the first time, an exothermic (liquid-expanded to condensed) and an endothermic (between two condensed phases) transition are observed during compression. At low temperature, Sur-PC-Sur (1) forms a condensed phase $\mathrm{LC}_{1}$ that is dominated by the intermolecular hydrogen bond network of the urea moieties. The optimization of the lateral headgroup-headgroup arrangement leads to a very high chain tilt angle of $52^{\circ}$. At higher temperatures and during compression, presumably the hydrogen bond network is broken allowing a lower chain tilt angle of $35^{\circ}$. This high change in membrane thickness violates the two-dimensional Clausius-Clapeyron assumption that changes in membrane thickness during a phase transition are negligible. Therefore, in this particular case the lateral molecular area has to be replaced by the molecular volume. 


\section{Funding sources}

Part of this work has been financed by the Swiss National Science Foundation (Grant PP00P2_166209) and the National Centers of Competence in Research - Chemical Biology as well as Bio-inspired Materials.

\section{Conflicts of interest}

The authors declare no conflicts of interest.

\section{Acknowledgements}

Parts of this research were carried out at DESY, a member of the Helmholtz Association (HGF). The authors thank Irina Berndt and Ingrid Zenke (MPI-KGF Potsdam) for help with isotherm and SAXS/WAXS experiments, as well as the PSI EM Facility for electron microscopy. This work has been supported by the University of Fribourg and the Swiss National Science Foundation through a stipend professorship to A. Z. and through the National Centers of Competence in Research (NCCR) in Chemical Biology and Bio-inspired Materials.

\section{References}

1 J. N. Israelachvili, Intermolecular and Surface Forces, Academic Press, San Diego, 3rd edn, 2011.

2 J. N. Israelachvili, D. J. Mitchell and B. W. Ninham, J. Chem. Soc., Faraday Trans. 2, 1976, 72, 1525-1568.

3 M. Ramanathan, L. K. Shrestha, T. Mori, Q. M. Ji, J. P. Hill and K. Ariga, Phys. Chem. Chem. Phys., 2013, 15, 10580-10611.

4 R. N. A. H. Lewis and R. N. McElhaney, in The Structure of Biological Membranes, ed. P. L. Yeagle, CRC Press, Boca Raton, 2012, vol. 1, ch. 4, pp. 19-90.

5 G. Büldt and R. Wohlgemuth, J. Membr. Biol., 1981, 58, 81-100.

6 A. Seelig and J. Seelig, in Encyclopedia of Physical Science and Technology, ed. R. A. Meyers, Academic Press, New York, 3rd edn, 2003, vol. 9, pp. 355-367.

7 P. Mattjus, R. Bittman and J. P. Slotte, Langmuir, 1996, 12, 1284-1290.

8 G. Brezesinski, A. Dietrich, B. Struth, C. Böhm, W. G. Bouwman, K. Kjaer and H. Möhwald, Chem. Phys. Lipids, 1995, 76, 145-157.

9 E. N. Serrallach, R. Dijkman, G. H. de Haas and G. G. Shipley, J. Mol. Biol., 1983, 170, 155-174.

10 B. Rattay, G. Brezesinski, B. Dobner, G. Förster and P. Nuhn, Chem. Phys. Lipids, 1995, 75, 81-91.

11 J. L. Slater and C.-H. Huang, The Structure of Biological Membranes, CRC Press LLC, 2nd edn, 2005, ch. 3, pp. 121-145.

12 G. Pabst, S. Danner, S. Karmakar, G. Deutsch and V. A. Raghunathan, Biophys. J., 2007, 93, 513-525.

13 M. N. Holme, I. A. Fedotenko, D. Abegg, J. Althaus, L. Babel, F. Favarger, R. Reiter, R. Tanasescu, P.-L. Zaffalon, A. Ziegler, B. Müller, T. Saxer and A. Zumbuehl, Nat. Nanotechnol., 2012, 7, 536-543.

14 I. A. Fedotenko, C. Stefaniu, G. Brezesinski and A. Zumbuehl, Langmuir, 2013, 29, 9428-9435.
15 A. Weinberger, R. Tanasescu, C. Stefaniu, I. A. Fedotenko, F. Favarger, T. Ishikawa, G. Brezesinski, C. M. Marques and A. Zumbuehl, Langmuir, 2015, 31, 1879-1884.

16 H. Noguchi, Phys. Rev. E: Stat., Nonlinear, Soft Matter Phys., 2003, 67, 041901.

17 N. Korin, M. Kanapathipillai and D. E. Ingber, Isr. J. Chem., 2013, 53, 610-615.

18 T. Saxer, A. Zumbuehl and B. Müller, Cardiovasc. Res., 2013, 99, 328-333.

19 D. Mellal and A. Zumbuehl, J. Mater. Chem. B, 2014, 2, 247-252.

20 N. Korin, M. J. Gounis, A. K. Wakhloo and D. E. Ingber, JAMA Neurol., 2015, 72, 119-122.

21 M. Epshtein and N. Korin, J. Biomech., 2017, 50, 217-221.

22 J. Wang, J. A. Kaplan, Y. L. Colson and M. W. Grinstaff, Adv. Drug Delivery Rev., 2017, 108, 68-82.

23 F. Neuhaus, D. Mueller, R. Tanasescu, S. Balog, T. Ishikawa, G. Brezesinski and A. Zumbuehl, Angew. Chem., Int. Ed., 2017, 56, 6515-6518.

24 C. Stefaniu, P. L. Zaffalon, A. Carmine, Q. Verolet, S. Fernandez, T. A. Wesolowski, G. Brezesinski and A. Zumbuehl, Langmuir, 2015, 31, 1296-1302.

25 P. L. Zaffalon, V. D’Anna, H. Hagemann and A. Zumbuehl, RSC Adv., 2013, 3, 7237-7244.

26 I. A. Fedotenko, P. L. Zaffalon, F. Favarger and A. Zumbuehl, Tetrahedron Lett., 2010, 51, 5382-5384.

27 P. Garidel, Phys. Chem. Chem. Phys., 2002, 4, 1934-1942.

28 P. Garidel, Phys. Chem. Chem. Phys., 2006, 8, 2265-2275.

29 D. Marsh, Handbook of Lipid Bilayers, CRC Press, Boca Raton, 2nd edn, 2013.

30 C. Stefaniu and G. Brezesinski, Adv. Colloid Interface Sci., 2014, 207, 265-279.

31 F. Bringezu, B. Dobner and G. Brezesinski, Chem. - Eur. J., 2002, 8, 3203-3210.

32 V. M. Kaganer, H. Möhwald and P. Dutta, Rev. Mod. Phys., 1999, 71, 779-819.

33 J. L. R. Arrondo, F. M. Goni and J. M. Macarulla, Biochim. Biophys. Acta, 1984, 794, 165-168.

34 C. Stefaniu, G. Brezesinski and H. Möhwald, Soft Matter, 2012, 8, 7952-7959.

35 C. R. Flach, J. W. Brauner and R. Mendelsohn, Biophys. J., 1993, 65, 1994-2001.

36 M. N. Antipina, B. Dobner, O. V. Konovalov, V. L. Shapovalov and G. Brezesinski, J. Phys. Chem. B, 2007, 111, 13845-13850.

37 A. I. Kitaigorodskii, Organic Chemical Crystallography, Consultants Bureau, New York, 1961.

38 C. A. Schneider, W. S. Rasband and K. W. Eliceiri, Nat. Methods, 2012, 9, 671-675.

39 J. Schindelin, I. Arganda-Carreras, E. Frise, V. Kaynig, M. Longair, T. Pietzsch, S. Preibisch, C. Rueden, S. Saalfeld, B. Schmid, J. Y. Tinevez, D. J. White, V. Hartenstein, K. Eliceiri, P. Tomancak and A. Cardona, Nat. Methods, 2012, 9, 676-682.

40 J. Schindelin, C. T. Rueden, M. C. Hiner and K. W. Eliceiri, Mol. Reprod. Dev., 2015, 82, 518-529.

41 R. Tanasescu, M. A. Lanz, D. Mueller, S. Tassler, T. Ishikawa, R. Reiter, G. Brezesinski and A. Zumbuehl, Langmuir, 2016, 32, 4896-4903. 